\title{
Individuating processes
}

\author{
John Pemberton
}

\section{Introduction}

Recent years have seen the development of a view that at least some entities that are widely taken to be individuals are processes, perhaps most notably biological organisms (See e.g. Dupré 2013, Bapteste \& Dupré 2013, Dupré this volume). But what are processes? How should we individuate them?

One way forward to address these questions would be to appeal to an existing metaphysical view of individuals, and to seek to impose this on processes. But, as the brief sketch in Section 4.1 shows, metaphysics encompasses a divergent and complex range of view concerning what it is to be an individual. To choose any one of these views is to make a choice and commitment, and for many this may seem too quick.

I propose proceeding in more modest steps. In the first step, I set aside metaphysics to explore the precise knowledge of processes achieved by practitioners and philosophers of science focusing on successful practice areas (e.g. biology, chemistry, engineering, physics), with the aim of distilling and articulating a prevailing view of processes adopted within these areas. In the second step, I sketch some of the accounts of individuals proposed by metaphysics. By juxtaposing these metaphysical views of individuals with the view of processes distilled from science, the paper aims to identify the tensions between these perspectives, and hence to cast light on the nature of processes and how they might be individuated. ${ }^{1}$

Section 2 undertakes this first step: distilling a characterisation of processes employed in the sciences. A consideration of many examples of the careful and specific accounts of processes developed in the sciences suggests that processes are taken to involve parts acting together (at each stage) to bring about the next stage of the process. In addition to how the process is at each stage (e.g. the spatial

\footnotetext{
${ }^{1}$ This approach aims to follow the recommendations of Guay \& Pradeu 2016 Chapter 1 and the longstanding principles of the Stanford School, and is congruent with the programme to develop a 'metaphysics of science' (see e.g. Mumford \& Tugby 2013).
} 
arrangement of parts at that stage), we may characterise how the process changes across stages, and how the process acts to self-maintain across each stage, i.e. an account of how it survives and hence exists (its existence is not taken to be just a brute fact). Moreover, many processes may often be intimately connected with larger processes of which they are a part and have roles in maintaining this larger contextual process, so that the nature of the context in which it occurs and the role(s) it plays within this larger context may often provide further useful characterising criteria of a process. There is thus a rich range of criteria that may be used to characterise processes.

The metaphysical discussion of individuals which seems potentially most helpful here is not focused on processes directly, but on individual things (sometimes termed substances or objects) ${ }^{2}$. In order to compare processes with things, I restrict (within this initial investigation) the focus to entities which are plausibly both processes from the perspective of science and things from the perspective of metaphysics. As discussed in Section3, this paper supposes that at least some entities fall in to this overlap, and suggest that perhaps many may do (e.g. perhaps organisms, parts of organisms, atoms, molecules, bundles, artefacts).

Section 4.1 adumbrates the broad range of metaphysical views concerning what it is to be an individual (thing) in order to make explicit the divergence of views and the key themes involved. This divergence of view presents a challenge for undertaking the required comparison with processes. To facilitate progress on this comparison, I articulate a single simple exemplar of these metaphysical views: an individual is taken to satisfy E. J. Lowe's identity and unity conditions (E. J. Lowe 2016, p59), and to be wholly characterised by a list of (admissible) properties. This simple exemplar view captures central aspects of many popular metaphysical account of individuals, such as bundle accounts and bare particular accounts. Comparison of this exemplar view with the view of processes distilled from science (in the context of the key metaphysical themes concerning what it is to be an individual which have been identified) are a first achievable step undertaken by this paper. This initial comparison, undertaken in Section 4, does identify interesting tensions between these perspectives.

Section 5 draws some provisional conclusions: Until tensions with the metaphysical account of individuals are better resolved, it seems reasonable to choose to use methods for individuating processes which have respect for the rich account of processes reflected in the practices of science. Respecting such a processual view may license recognising both processes and certain of their parts (e.g. organism / cell, biofilm / bacterium, species / organism) as both being valid unities (e.g. sufficiently robust processes).

\footnotetext{
${ }^{2}$ I have not found salient material on individuation within the process metaphysics literature (see e.g. works by Whitehead, Rescher, Seibt) - I do not here seek to employ insights from this domain.
} 


\section{Processes in science}

A review of text books, articles and research papers across the sciences reveals the widespread use of diagrams which represent the spatial layout of the parts of things or processes which are of interest. Often these diagrams feature arrows indicating how the layout changes over time, or perhaps a sequence of such diagrams which denote the stages of such change. Typically, accompanying text describes this changing of the arrangement and the properties of the parts through time, and how it is that the parts bring about this changing.

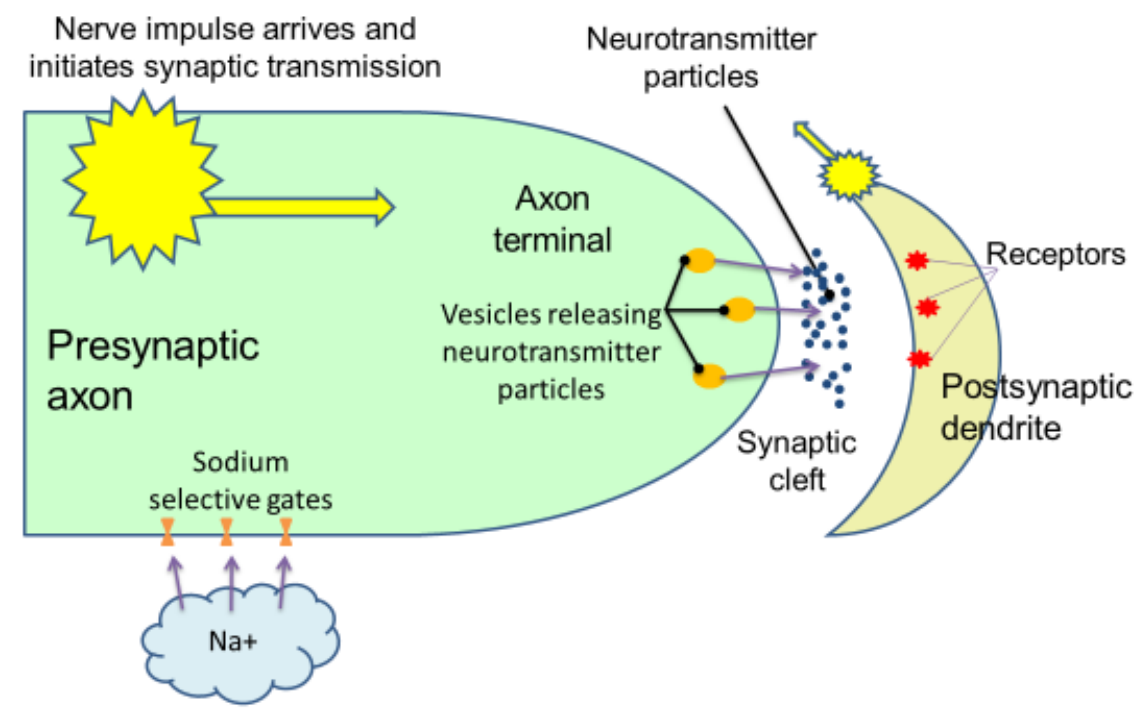

Figure 1: Diagram of synaptic transmission ${ }^{3}$

As an example, Figure 1 shows the layout of some of the key parts involved in synaptic transmission which is similar to diagrams used in many biology text books such as Bear et al 2007 (Figure 2.15), whose accompanying text explains:

When a nerve impulse arrives in the presynaptic axon terminal, neurotransmitter molecules are released from synaptic vesicles into the synaptic cleft. Neurotransmitter then binds to specific receptor proteins causing the generation of electrical or chemical signals in the post-synaptic cell. (Bear et al 2007, 39)

Other works focus on certain of the parts shown in Figure 1, and depict diagrammatically the acting together of their more detailed parts - Südhof and Rizo, for example, use a series of spatial diagrams to explicate the vesicles and the processes in which they engage (Südhof and Rizo 2011, Figures 1-5). Other texts focus on numerical measurements of aspects of such processes, for example

\footnotetext{
${ }^{3}$ All figures in this paper are by the author.
} 
Eijkelkamp et al present alongside a spatial diagram representing the operation of a sodium gate, a graph depicting the magnitude of the potential across the gate over time during the passage of a nerve impulse, i.e. action potential (Eijkelkamp et al 2010, Figure 1).

This example is representative of a vast number of other examples across the sciences. A common feature of the processes explicated in such diagram-based accounts is the acting together of parts within some arrangement at each stage, to bring about a next stage - this is consistent with the minimal representation of a process shown in Figure 2.

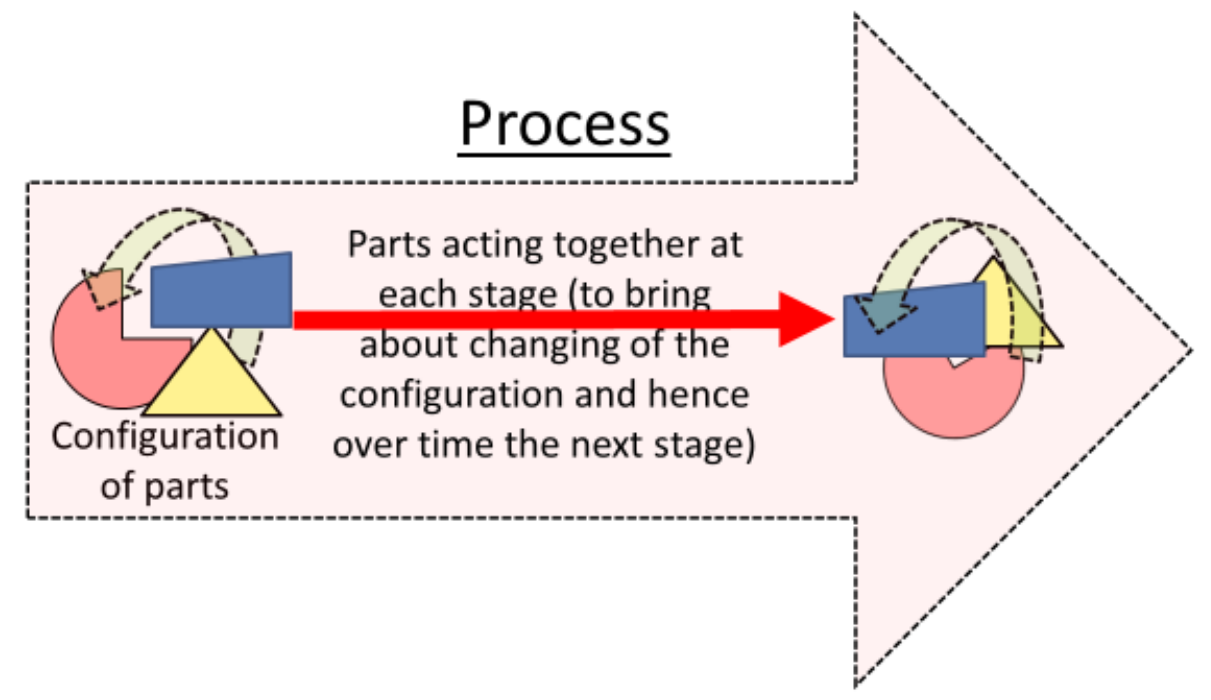

Parts (as pictured by science):

- Are spatially located (i.e. physical)

- Can act with certain other parts

Figure 2: A process

The view that much science is centrally concerned with such configurations of interacting parts is supported by many philosophers of science, perhaps most notably the new mechanists, who take mechanisms to be widely occurring ${ }^{4}$. Such mechanisms are taken to involve parts which are spatially organised acting together to exhibit patterns of change (sometimes referred to as activities or behaviours of the mechanism) (Machamer, Darden \& Craver 2000, Bechtel \& Abrahamsen 2005, Glennan 2002, Illari \& Williamson 2012). The views of such philosophers of science have particular salience as they are typically based on careful and extensive observation of examples from the practice of science. These examples, which generally feature processes in the sense of Figure 2, are taken to exhibit patterns of change associated with a mechanism, and are often explicitly recognised as processes. $^{5}$

\footnotetext{
${ }^{4}$ Some new mechanists take mechanisms to be widely occurring throughout the sciences (e.g. Glennan forthcoming), others focus on narrower areas and take mechanisms to occur widely within their areas of focus (e.g. Darden 2006, Craver 2007).

${ }^{5}$ Although many accounts of mechanism do explicitly embrace processual characteristics, they fall short of providing an adequate account of the link between the arrangement of parts and the change
} 
I take Figure 2 as representing at least many of the processes studied by science, and adopt it as the starting point for the scientific picture of processes.

\subsection{Characterising processes}

This section builds on the characterisation of a process in Figure 2 by identifying the various characteristics that processes are commonly taken to have by those who make use of processes across the various fields of scientific practice. The characterising criteria identified are not intended as a definitive list, but as a reasonable starting point which others might choose to amend according to their differing views or purposes.

Figure 2 makes clear that the processes posited in the sciences are time-extended: they involve a changing (or perhaps stasis) of a configuration over time. The first 3 of the following criteria follow straightforwardly from this picture in Figure 2.

\section{a) The nature of the parts at each stage}

The elements of the spatial diagrams used in science, noted above, are typically shapes or pictures representing the parts of the process - i.e. the spatially located entities which act together with the other parts at each stage to give rise to the process. The diagram typically represents some stage of the process (although arrows or other pictorial devices may be used to suggest the pattern of change over time) - and hence the parts which are present and perhaps acting at that stage. The nature of the parts varies from science to science: in biology perhaps neurons, vesicles, neurotransmitter particles, as in Figure 1. In astronomy familiar spatial diagrams include those with force arrows indicating the mutual gravitational attracting (acting together) of stars (planets) and their planets (moons) and the resultant elliptic orbits - here parts include stars, planets and moons. In chemistry parts may include electrons, protons, molecules; in engineering perhaps cogs, spark plugs, cylinders. In each case the parts are typically entities familiar to the science concerned.

\section{b) The spatial arrangement of the parts at each stage}

By choosing the duration of a salient stage as sufficiently brief, we may typically be able to limit the change of the configuration during that stage so as to be able to characterise the arrangement of parts (i.e. their spatial locations, orientations and velocities) at that stage using a single snapshot spatial diagram. Sometimes a diagram may show schematically the spatial layout of differing parts at differing times, perhaps using arrows to indicate the time sequencing (as in Figure 1). In

which occurs, e.g. in the Machamer et al account the link between entities and activities - see Pemberton 2011. Cartwright (recently with Pemberton) by embracing powers does provide a basis for such a link within nomological machines, which may be understood as a version of mechanisms (Cartwright \& Pemberton 2012, Pemberton \& Cartwright 2014, Chen 2017). 
engineering, the spatial arrangement of parts may typically be specified by use of a blueprint (which may also generally specify the nature the parts), which may be used as the basis for constructing the configuration, and hence instantiating the relevant process. Similarly, for certain laboratory experiments, the initial spatial layout of pieces of laboratory equipment and entities under study may be represented in a diagram - when the layout is instantiated the aim may typically be to produce some repeatable process involving the entity under study, i.e. a run of the experiment. In other sciences (such as astronomy, biology, geology) the processes in focus are typically found in nature - the diagrams may show the spatial layout of typical processes of the relevant kind at salient stages.

\section{c) The nature of the change a process exhibits across certain stages}

The swinging of a pendulum might be described as U-shaped or perhaps as exhibiting a stopped-slow-fast-slow-stopped pattern. Other processes may be smooth, intermittent, or explosive; or perhaps give rise to spherical, circular or linear spatial patterns of change. In Figure 1 the rough pattern of change over time is indicated by the arrows and explicated further by the text.

We may note that the temporally-extended nature of processes (represented in Figure 2) leads to two differing senses in which the term 'process' is often used. Sometimes the changing which occurs may be in focus - i.e. criteria (c), e.g. the swinging of a pendulum, the beating of a heart, a run of some experimental set-up in the laboratory. This changing occurs over time and this sense of the term 'process' might be thought of as somewhat abstract. Alternatively, we might have in focus the configuration of parts which are acting at each stage, e.g. the swinging pendulum, the beating heart, the acting parts of the experimental set-up ${ }^{6}$. I take a process to embrace both these notions - this is reflected in the recognition of criteria (a), (b) and (c).

\section{d) Beginning-to-end / homogenous on-going / heterogeneous on-going}

Some types of processes, beginning -to-end processes say, run through from a certain type of starting configuration to a certain type of ending configuration via a series of well-defined stages - perhaps over some rough given timescale. The firing of a neuron, for example, perhaps starts with neurotransmitter particles binding with ligand-gated neuroreceptors, hence opening certain ion channels. The movement of ions across the neuronal membrane which this licences, may result in an increased potential across the membrane, and hence the opening of nearby voltage-gated ion channels, which may in turn lead to a cascade of voltage-gated ion channels opening along the neuron, and then to the opening of vesicles which release neurotransmitter particles.

Again, when a coin is inserted in a drink vending machine a process of well-defined stages may ensue which results in a drink in the output bin.

\footnotetext{
${ }^{6}$ In Carl Craver's terms, 'the $\psi$-ing S' rather than 'the $\psi$-ing of S' (Craver, 2007, 7).
} 
Beginning-to-end processes exhibit heterogeneity: perhaps differing parts act at differing stages, or perhaps parts act in differing ways at differing stages. The ligandgated neuroreceptors feature at the start of the process of the neuron firing, whilst voltage-gated ion channels and vesicles feature later on. The coin inserted in the vending machine perhaps depresses a lever which turns various cogs leading on to the acting of different parts which release the cup and liquid.

Other processes (although they do generally have a beginning and an end) may be thought of as on-going. This is typically the case with processes which exhibit a high degree of homogeneity. As an example of such a process, consider a water molecule.

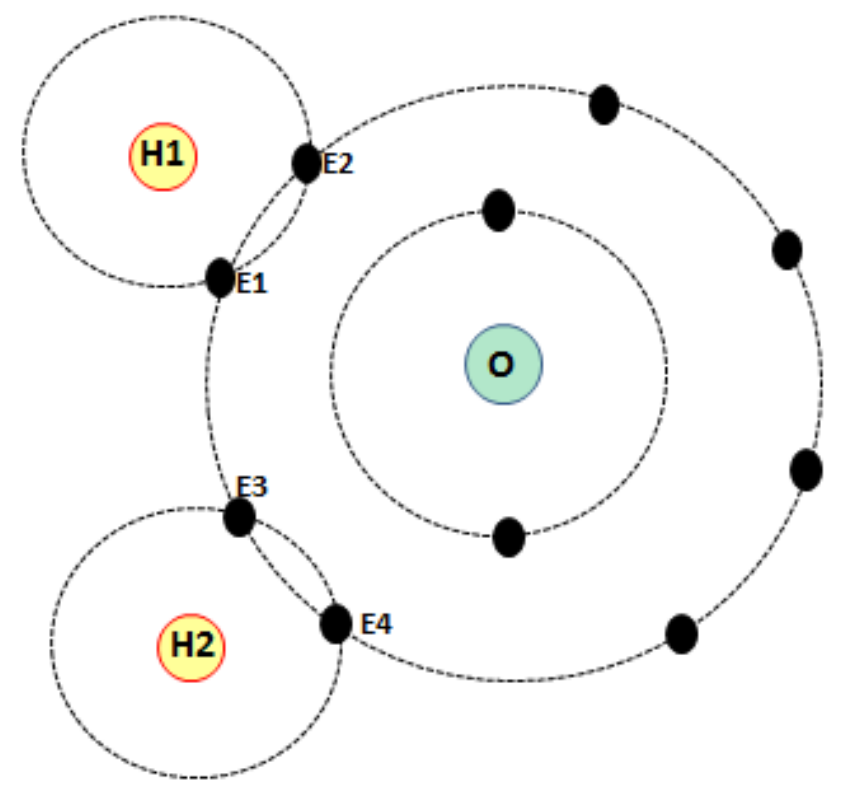

Figure 3: A water molecule - an example of a process

On the standard scientific account, the parts of the water molecule are protons, oxygen nucleus and electrons arranged along the lines shown in Figure 3 (see e.g. Keller 2013 - cover diagram). Within this configuration, the parts exert (and experience) basic forces of physics on (due to) other parts - perhaps most notably electrostatic forces associated with charge. ${ }^{7}$ This acting together of the parts ${ }^{8}$ in this way, may be understood as a process which continues through time in a way which is self-sustaining. This process seems to exhibit a high degree of homogeneity: the same parts are acting at each stage and acting in the same sort of way (although perhaps the strength and direction of the basic forces may vary over time).

Bundles, as characterised by Aristotle for example (e.g. Metaphysics V.6.), may also be taken to be examples of homogeneous on-going processes. Consider for example

\footnotetext{
${ }^{7}$ This paper does not attempt to address the complex and divergent views prevalent within the metaphysics of quantum mechanics - the classical view suffices for the purposes of this paper.

${ }^{8}$ The exerting of a force and the experiencing of a force are how the actings of the parts (e.g. masses, charged bodies) may be understood in this context.
} 
a bundle of sticks bound by some binding such as string. When roughly static, the binding gives rise to forces which hold the bundle together, whilst the sticks play their role by pushing against each other and the binding so as to form a stable physical unit - the same parts and roughly the same forces act homogeneously at each stage. When external forces act, e.g. when the bundle of sticks is kicked, the binding forces within the bundle react - they increase so as to prevent the sticks flying apart, for example. ${ }^{9}$ In this reactive way, bundles are effective at selfmaintaining, and may be viewed as a common, if somewhat prosaic, form of process. At the micro level bindings may be atomic or molecular bonds, so that the bundles may then be graphite rods, diamonds, lumps of copper, rocks, drops of water, etc. At the macro level forms of binding may include tying, gluing, nailing, bolting, interlocking of congruent shapes, containing by a wall, linking by tissue / fibre, etc. Examples of macro bundles may include bundles of sticks, packets of biscuits, broken (or perhaps non-functioning) mechanisms, and dead organisms.

By contrast, consider an example from engineering: a process which is exhibited by an artefact such as a motorbike. This process transitions through differing stages involving the acting of differing parts at each stage: the sparking of the cylinder by the spark plug, the exploding of hydrocarbons, the driving down of the piston, the opening of the exhaust valve, the emitting of the exhaust gasses, the closing of the exhaust valve, the moving upwards of the piston, and the admission of new hydrocarbons. In this sense, the process exhibits a high degree of heterogeneity. By ensuring that the process arrives at roughly the same configuration as it started, the process is rendered cyclical, and hence on-going - a heterogeneous on-going process we may say.

Organisms may involve a processual transition through developmental stages, e.g. seed, young and mature organism; where each stage exhibits multiple life processes (e.g. mitosis, respiration, digestion, photosynthesis, self-repair, etc.). The view has therefore developed that organisms are processes - if so, then these processes too exhibit heterogeneity, but in a vastly more complex way than machines. The differing parts of the organism may act in differing ways at differing stages to selfmaintain the organism and perform desired functions. Many of the sub-processes of the organism (e.g. the firing of a neuron, the contraction of a muscle, the digestion of a meal) might be taken to have a beginning-to-end character. But the appropriate coordination of such sub-processes ensures that the organism as a whole is on-going - it is another form of heterogeneous on-going process.

This 3-fold classification of processes into beginning-to-end / homogeneous on-going / heterogeneous on-going might be further refined (e.g. to capture distinctions between mechanical and organic heterogeneous on-going processes) or developed it is intended as indicating a start-point for a classificatory criterion.

\section{e) Originating-things}

\footnotetext{
${ }^{9}$ Of course, a sufficiently hard kick may cause the bindings to break or sticks to escape the bindings, so that the bundle ceases to be.
} 


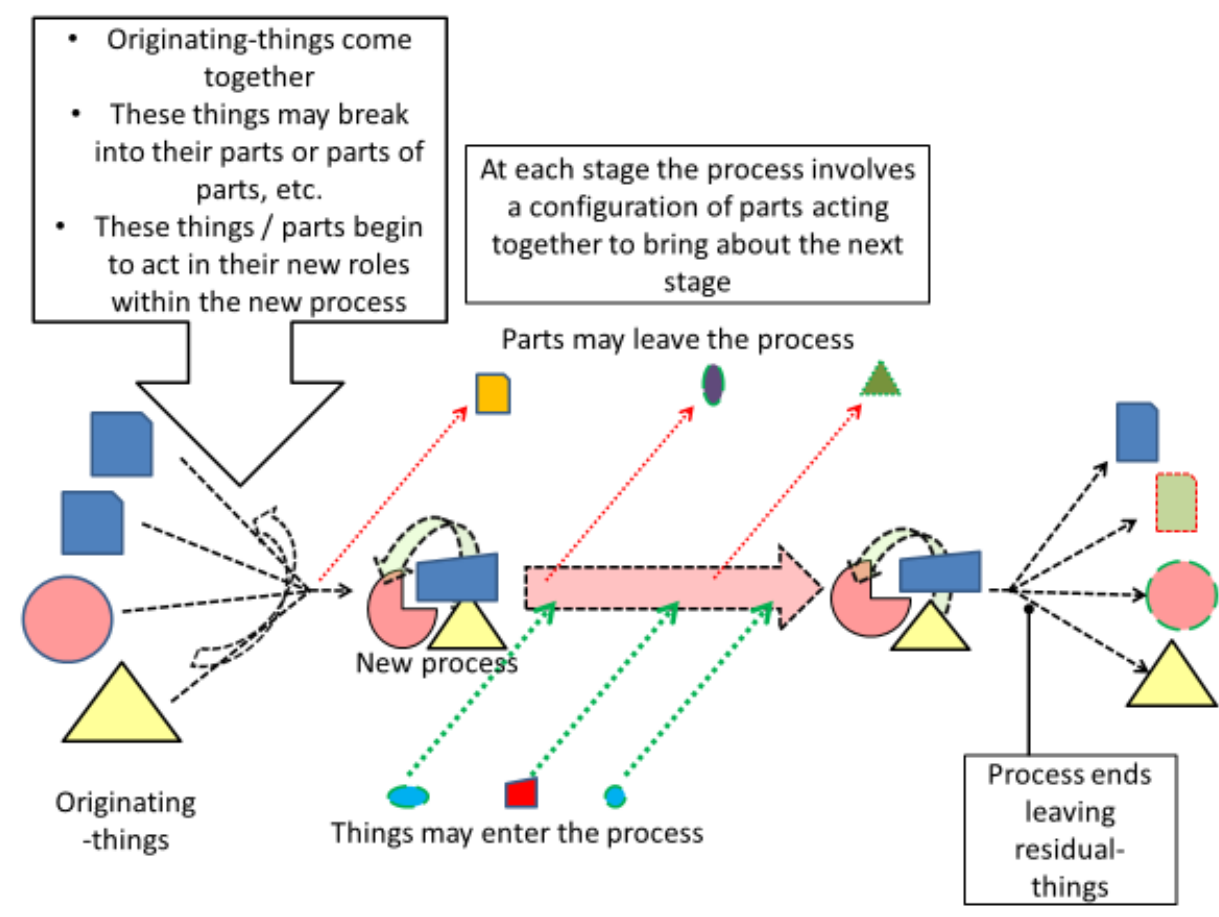

Figure 4: Originating-things come together to form a new process - and then things may join and leave the process

Sometimes we may characterise a process according to the things which came together to give rise to that process, the originating-things say. Originating-things may or may not remain as identifiable unities within the new process.

For example, we might take the cogs, cylinders and spark-plugs used to build a motor-bike as the originating-things of that bike - these originating-things do typically remain as identifiable parts of the motorbike. In biology we might take the originating-things of a certain mule to be the gametes from the donkey and the horse which fused to form the zygote which was the earliest stage of this mule. The story of these gametes after their fusion is complex - it does not seem that these originating-things remain as identifiable unities within the mule longer term. Certain eggs may be amongst the originating-things of a cake - these originating-things do not remain as unities within the cake.

f) The nature of the things / parts which join or leave the process at each stage

Things may both enter and leave a process at each stage (as illustrated in Figure 4). For example, hydrocarbon fuel (motorbike) or nutrients (organism) may enter a process, whilst exhaust gasses or urea may leave a process. Processes are often characterised by the nature of the things which join or leave, for example organisms may be characterised as herbivores or carnivores (according to the nature of food taken in by the organism); and similarly engines as diesel or petrol - or perhaps according the rate of emission of certain polluting gasses.

g) Residual-things 
When a process comes to an end there may typically be some things which are left, residual-things say. Following the death of an organism, i.e. the ending of its life process, a corpse remains (initially at least). When an experimental set-up is run in the laboratory, perhaps a chemical experiment, the process which occurs may give rise to characteristic products and residues.

\section{h) How the process self-maintains at each stage}

The widespread supposition of science is not that the sequence of stage configurations of a process is inexplicable, but rather that the nature of the parts and their configuration at each stage brings about the next stage. (Where the process exhibits a single on-going configuration, it may be supposed that the acting of the parts at each stage maintain that configuration.) Typically, an account of how the parts act together at each to bring about changing, and hence the next stage of the process, is available for each of the spatial diagrams of the parts illustrated and discussed above. For example, the approaching nerve impulse (action potential) of the neuron in Figure 1, opens sodium selective gates allowing movement of sodium ions and hence the local changing of potential difference across the wall of the neuron, which underwrites the movement of the action potential along this part of the neuron. At the axon terminal, the action potential opens the vesicles releasing neurotransmitter particles which cross the synaptic cleft and dock with receptors, etc. It is supposed that a detailed story is available (even if not yet discovered) about the acting of parts at each stage, and how these lead on to the next stage.

How a process self-maintains across each stage may often be a useful criterion for classification. In the case of homogeneous processes, the process may self-maintain in largely the same way at each stage - a hydrogen atom, for example, self-maintains at each stage through the acting together of a proton and an electron, i.e. the mutual exertion of basic forces of physics. A bundle self-maintains through binding forces which are similar at each stage (although these forces may increase reactively when the bundle is stressed).

For heterogeneous processes, the account may typically vary across stages. A fire self-maintains at each stage by the oxidation of hydrocarbons releasing energy to maintain a high localised temperature and hence the release of more flammable gasses - but the parts (e.g. firelighter, kindling, logs, coal) acting at each stage may vary. For an engineered machine which transitions through a dynamic locus of configurations before perhaps returning (more or less exactly) to some starting ${ }^{10}$ configuration (such as motorbikes, cisterns, toasters, pendulums), an adequate account might reference how the process self-maintains through each different stage of the locus of configurations.

\section{i) Role in a larger process}

\footnotetext{
${ }^{10}$ As the locus of configurations exhibits a cycle, we might choose to ascribe any point in the cycle as the starting configuration, but where such mechanisms require a human action (e.g. pulling a chain, pushing a button, depressing a lever) to initiate a cycle, it is most natural to choose the rest configuration before this human action as the starting configuration.
} 
A key (which might be viewed as a shaped-bundle type process) can engage in a process of lock-opening when it is in a suitable configuration with a lock and a person, e.g. it is inserted in the lock and rotated by the person (a person-turning-keyin-lock process, say). We might ascribe to the key (qua shaped-bundle process) the role of opening the lock. We might also ascribe this role to the person-turning-keyin-lock process but in a different sense: this process may end with the lock being open.

In the biological context, many biochemical structures, such as proteins, have keylike (or lock-like) characteristics. ${ }^{11}$ A protein which is an enzyme, for example, may have a role as catalyst in some particular biochemical reaction - typically the reactants bind with the protein's active site whose spatial molecular structure and charge pattern lowers the energy requirement for the reaction (see e.g. Copeland 2000, especially 1.4 and 1.5 ). Other possible roles for proteins include transporter, inhibitor, and binding agent, for specific molecules or reactions. The role, or function, of a protein has been embraced as a crucial taxonomic criterion within extensive recent work in this area (see e.g. Wu et al 2004).

Many other parts of organisms (and the processes in which such parts engage) may also be classified according to their role. For example, a heart pumps blood, a kidney cleans blood. In engineering, a cog in the context of a bicycle might be classified as a drive sprocket - i.e. has the role of transmitting drive via a chain.

In some cases, the role performed by a part is central to the identification of that part and the salient process in which it engages. When a neuron is transmitting an action potential, certain configurations of polypeptide strings located in and around the walls of the neuron act together to open or close a pore in the lining of the neuron, hence allowing or inhibiting the passage of sodium ions across the neuron wall. It is this role within the higher-level process of neuron-firing that helps to license recognition of the unity of this process (i.e. the acting together of this configuration of polypeptide strings), which may be referred to as the opening / closing of a sodium selective gate, and hence the unity of the sodium selective gate itself.

\section{j) The context in which the process occurs}

Processes may also be classified according to the context in which they occur, e.g. stellar nucleosynthesis as occurring within a star, certain biochemical process as intracellular, and action potential transmission as neuronal.

One reason a process may be classified according to such a context is that the process requires a configuration of certain types of parts, and that such configurations only obtain in certain restricted types of context. Another reason is that a process is recognised as performing a role in a larger context (as in (i) above) -

\footnotetext{
${ }^{11}$ This is not to advocate simplistic key-in-lock theories of e.g. enzyme operation.
} 
the larger context must then obtain: for a heart to pump blood it must be located within a suitable circulatory system. The (pumping) heart may be classified as part of this circulatory system.

On other occasions certain features of a context may facilitate a process in other ways. For example, the operation of many proteins within intracellular processes depends critically on their being correctly folded and spatially configured (and not merely having a certain topological structure). The achievement of such suitable folding and spatial configuration may rely upon chaperone proteins, so that the context must feature such proteins for the relevant processes to occur. ${ }^{12}$

\section{k) The phase-stages of a process}

As noted in criterion (d), processes vary considerably as to their degree of heterogeneity. Some processes exhibit such a high degree of heterogeneity across sequential stages that it may be unclear whether they should be treated as one process or a series of sequential processes. For example, an astronomic feature may have the stages diffuse nebula, main-sequence star, red giant, and white dwarf; an organism may have stages egg, caterpillar, chrysalis, and butterfly. I dub such radically differing stages phase-stages. Where a process is taken to exhibit such phase-stages, the nature of these phase-stages, and how they lead on from each other, may provide a further useful classificatory criterion.

This consideration of processes that are referenced within science identifies a rich framework of characterising criteria. Which of these types of criteria are useful in any given case will depend on the nature of the process which is in focus. Typically, a number of criteria of differing types may be helpful in establishing a suitable characterisation. Where a process may be taken to involve phase-stages, it may be appropriate to characterise each phase-stage using criteria of types (a) - (j).

Processes, on this characterisation, involve the acting together of parts at each stage. As noted in the discussion of Figure 4, the coming in to being of a process may involve the coming together of things which then act together as parts in the new process, as in the construction of a motorbike. The vastly more complex processes studied in biology may come in to being via the union of gametes, themselves vastly complex processes, and then prolonged development stages. Astronomic processes, such as stars, may involve the incremental coming together of massive bodies in a way which is different again.

\footnotetext{
${ }^{12}$ See Dupré 2010 for a discussion of the importance of context for biological entities.
} 


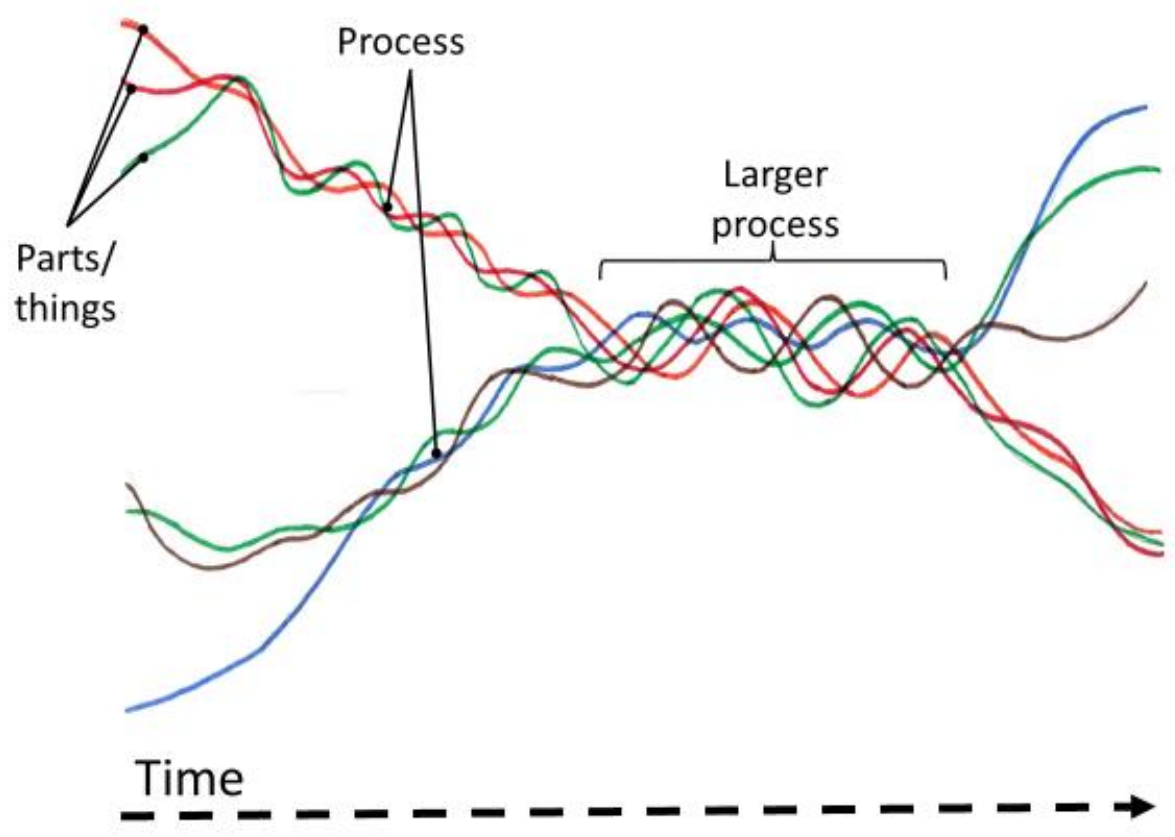

Figure 5: A picture of processes drawn from science

Figure 5 develops Figure 4 to illustrate this pictorially: things, perhaps themselves processes, may come together to form processes (represented by intertwining strands), which may in turn combine (perhaps via incremental development) to form larger processes. Each of the parts (represented in the picture by a single coloured strand) may itself be a process of smaller parts (an intertwining of smaller strands). As discussed above, originating things may or may not survive as unities within a new larger process.

\section{Individuating processes - preliminary comments}

An initial thought might be that careful characterisation of processes along the lines outlined above, together with a specification of time and place, might be sufficient for individuating processes. In the case of some well understood sufficiently macroscopic processes, such as a beating heart or firing neuron this may often be so. For example, we may be able to pick out and keep track of a particular neuron and identify particular processes, e.g. neuron firings, as those processes of neuron-firing type which occur in this particular neuron at certain particular times. Each firing might be recorded as having a certain action potential intensity and duration, and a plurality of firings might then allow, for example, for the typical range of intensities and durations of firings of this neuron to be estimated.

In other cases, individuation of processes is more challenging: If we take a leaf mould to be processual, is it one process or many processes? Is a certain butterfly the same process as its chrysalis? Is the forest fire the same fire as the flame of Harry's match which lit the cigarette whose glowing butt caused the fire? 
It is consideration of such more challenging questions that suggests enlisting the expertise of metaphysics concerning the nature of individuals and individuation. Metaphysics, however, is not typically focused on the individuation of processes (at least not explicitly), but its more common focus is individual substances or things. In order to bring to bear the insights of metaphysics concerning the nature of individual things, I propose to focus in this initial investigation on entities which may be viewed by science as processes and which are widely considered by metaphysics to be individual things - a plausible preliminary view of this overlap is indicated in Figure 6.

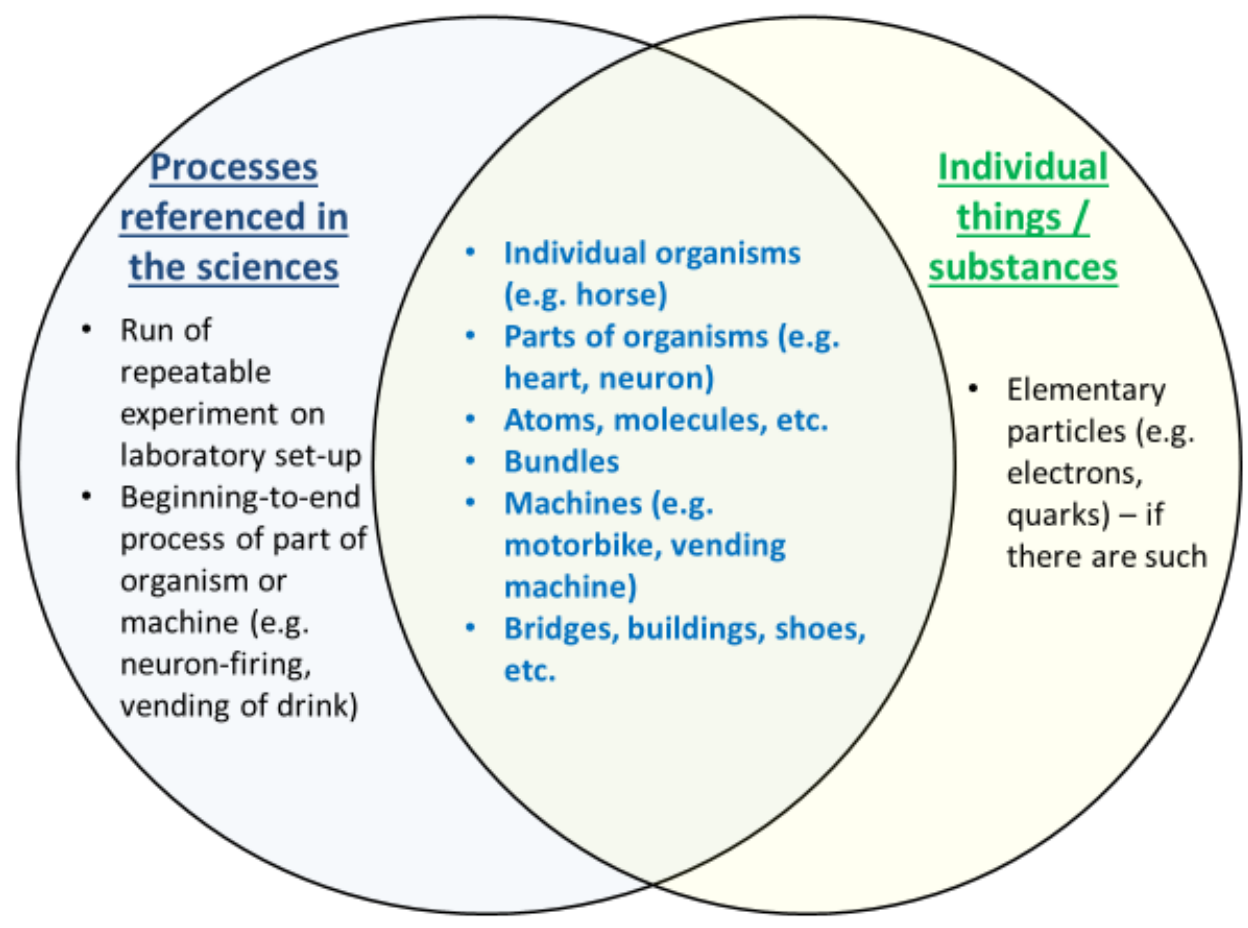

Figure 6: Entities that may be viewed by science as processes and by metaphysics as individual things - one possible view

If we take a string and attach one end to a bob and the other end to the top of a bench stand, and then set the bob swinging, we may have a simple pendulum, which we may take to be a process. If we now lie the stand-string-bob on its side on the bench it no longer swings - perhaps this apparatus is now a different type of process, maybe an organised bundle. If these are two differing processes, are they the same thing?

Similarly, we may ask: Is a beating heart understood as the process of the parts of the heart acting together over time, the same as a heart taken to be a thing, perhaps some compresent plurality of properties? Aristotle, for example, draws a clear ontological distinction between a heart acting in its bodily context and a disconnected heart, viewed as only homonymously a heart (Aristotle Metaphysics Z).

These are difficult questions which seem highly relevant to the topic of individuating processes. The strategy of this paper, as indicated in Section1, is not to address such questions directly from metaphysics, but rather to juxtapose a scientific view of 
processes and a metaphysical view of things as the basis for an initial exploration of the tensions between these views (this exploration is undertaken in Section 4). It is important to be clear that the Figure 6 is not presupposing an answer to such questions, but is merely identifying entities that are plausibly viewed by science as processes and by metaphysics as individual things.

It is a supposition of this paper that at least some entities fall into this overlap individual organisms, such as horses, are perhaps the most cited example. We have noted in Section 2 many processes referred to by the sciences and indicated the sense in which they may be taken to be processes. Many of these are also often considered to be individual things, e.g. parts of animals (e.g. hearts), atoms, molecules, bundles, fires.

Some processes are less plausibly things. For example, whilst an experimental set-up in the laboratory running from some starting configuration though to termination may generally be considered a well-defined process, it might not generally be taken to be a thing. Similarly, an action potential (neuron-firing), perhaps a beginning-toend process involving certain parts of a neuron cell, does not seem like a thing. Rather the neuron cell as a whole, a coordinated and complex multitude of processes which perhaps self-maintains in a way more similar to an organism, might more commonly be viewed as a thing. Machines seem similar in this respect to neurons: it is the vending machine which we might typically take to be a thing, rather than the beginning-to-end process of vending a drink.

Many seemingly static artefacts which are taken to be things, e.g. shoes, bridges, buildings, self-maintain their organised structure under conditions of use in a way that is similar to, but more precise than, bundles. Tall buildings, for example, must be designed to sway in the wind returning to equilibrium, bridges must contort as they accept load and then return to equilibrium as it is removed. Each of these artefacts involves the self-maintaining acting together of its parts and might reasonably be considered a process.

Are there things which are clearly not processes? If physics is right that there are some elementary particles - e.g. the particles of the Standard Model, such as electrons, muons and quarks - then it seems they do not involve the acting together of parts and hence they would be things which are not processes (in the sense explored here).

Although this discussion suggests that it is plausible that many (but not all) processes and many (but not all) things may fall into the overlap (as indicated in Figure 6), opinions on this may differ. The exact extent of the overlap is not crucial to the argument in the next section (although those who take it be much narrower may need to disregard some of the examples). 


\section{Comparing processes in science with individuals in metaphysics}

There are many widely differing views of individuals within metaphysics - it is not plausible to compare each of these against the picture of processes sketched in Section 2. The paper therefore adopts the following pragmatic approach. Firstly, some of the leading views of individuals in metaphysics are briefly recapitulated, so as to make explicit a rough adumbration of the range of views concerned and the key themes involved. Secondly, a single simplified exemplar metaphysical view of individuals from within this range is outlined. Although this is just one view, it reflects a consensus of many leading contemporary metaphysical views of individuals. Comparing this exemplar metaphysical view with the view of processes in science (focusing on processes which are plausibly individuals), provides an initial indication of the tensions between these perspectives. This is, of course, merely a first step to determining whether and how metaphysics may help with the individuation of processes which is achievable within this brief paper.

\subsection{Individuals in metaphysics - the divergence and complexity of views}

Aristotle supposes that metaphysics is the study of being qua being (Metaphysics, Book $\Gamma$ ), and at the very centre of this project is the explication of discrete individuals, substances, which have ontological priority. Aristotle rejects Plato's account of individuals as the co-instantiation of universals at spatio-temporal locations due, inter alia, to its failure to solve the nature-feature problem, i.e. to distinguish what a thing is from its accidental properties (see Scaltsas 1994, especially chapter 3 ) - and hence to provide an account of continuity through change. Individual organisms, such as horses or humans, are identified by Aristotle as paradigm examples of substances - and these are understood as involving both matter and form. But to achieve ontological priority, the substantial whole must be an unqualified unity of its matter and form - establishing this unity presents Aristotle with a challenge. His answer is the hylomorphism according to which matter is reidentified according to the substantial form so as to become unified as a whole which has no actual parts ${ }^{13}$.

Aristotle's project to identify substances is taken up by many generations of metaphysicians, who are often cautious of Aristotelian forms. Medieval philosophers, perhaps most notably Duns Scotus, posit haecceities: non-qualitative properties which are responsible for individuation and identity (Duns Scotus 1639). Leibniz posits monads as fundamental particulars which exhibit discrete individuality (see e.g. McCullough 1996). A long tradition posits bare particulars as the basis of ontological individuality (see e.g. Sider 2006).

\footnotetext{
${ }^{13}$ See Marmodoro (2013) for a lucid account and further references to the recent debate concerning Aristotle's hylomorphism.
} 
More recently, linguistic analytic philosophy has a differing motivation for positing discrete individuals, as Ruth Barcan Marcus explains:

The notion of an individual object or thing is an indispensable primitive for theories of meaning grounded in standard model theoretic semantics. One begins with a domain of individuals, and there are no prima facie constraints as to what counts as an individual except [each] must be distinct from every other and identical to itself (Marcus 1975, 39).

This logico-linguistic concept of individual perhaps finds its most well-known expression in Quine's dictum, often expressed as 'to be is to be the value of a bound variable' (see e.g. Quine 1948). The notion of individuality in play here does not appeal to any ontological criteria for demarcating individuals, such as spatial continuity, boundedness or other physical criteria which may often be salient within scientific contexts.

Central to a consideration of metaphysical accounts of individuals is their differing motivations for individuation:

1. One focus is picking out individuals at a particular time so as to separately identify one individual (Tom, say) from another (Dick, say). An individual which can be picked out as distinct from a sibling in this way satisfies what Lowe calls the identity requirement on individuality (E. J. Lowe 2016, 59). An electron within an entangled pairing may be an example of a putative individual which fails this requirement. It is often suggested (controversially) that Aristotle adopts matter as a principle of individuation: Tom is then distinct from Dick on account of differing constituent matter. Spatio-temporal location is another approach proposed to achieve such individuation, notably in the neo-platonic or Humean traditions.

2. Another issue is picking out the same individual (or different individuals) at differing times. Now it seems that matter is problematic as a principle of individuation: the matter of organisms (the leading contenders for substances) may change over time. For macroscopic individuals, in biology for example, spatio-temporal continuity may provide a practical criterion for resolving this challenge, but it is less clear this will do for the sorts of particles posited by contemporary physics.

3. A further important focus is determining whether a putative plurality of parts obtaining at some time constitute a single whole, i.e. a single individual constituted by the parts, or not (Van Inwagen 1990). This requirement to be one entity is what Lowe terms the unity requirement on individuality (E. J. Lowe 2016, 59). Parts are held to present a challenge for many metaphysical accounts of individuals as they are often deemed to threaten the unity of the whole. Aristotle, for example, supposes that there can be no actual parts within a substantial whole, only potential parts. 
Even this brief sketch of a handful of views makes clear that there are divergent metaphysical opinions on what it is to be an individual, and that picking out a single metaphysical view involves a choice and commitment.

\subsection{Exemplar metaphysical view of individuals}

The exemplar view supposes that an individual satisfies E.J. Lowe's unity and identity requirements for individuality, and that individuals can be wholly characterised by a list of admissible properties at each time (so that an individual may perhaps be taken to be a series of such property-defined entities at successive times). This exemplar view captures central aspects of metaphysical accounts according to which individuals are co-instantiation of platonic or other universals, bundles of properties, bare particulars with properties, collections of tropes, or co-locations of powers.

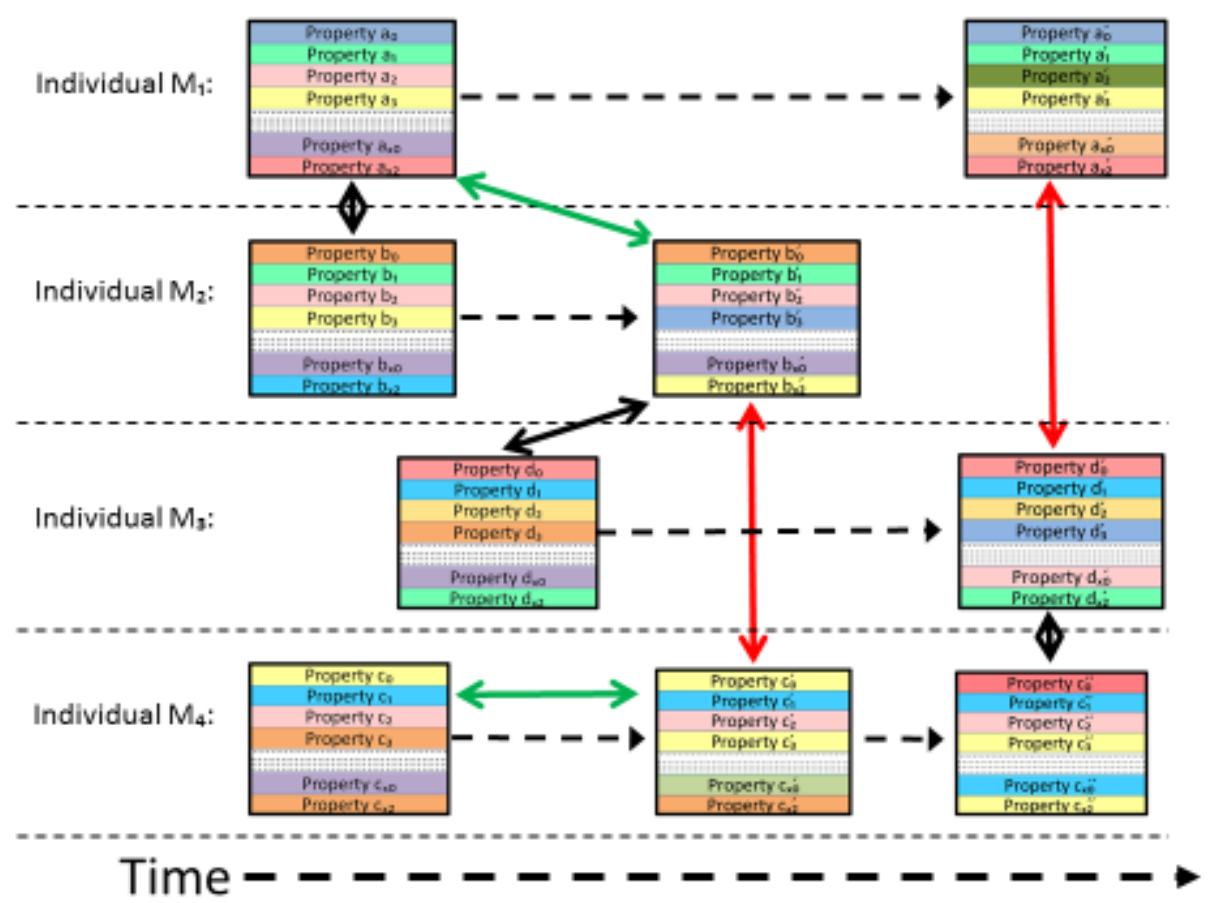

Figure 7: A picture of the exemplar metaphysical view of individuals

Figure 7 pictures this exemplar view: Each box, which is wholly characterised by a list of properties, represents an individual at some time. The horizontal dashed-lines represent the passage through time of these individuals. The boxes are pictured as separate and distinct - this reflects the individuals satisfying Lowe's identity requirement. No box has other boxes as parts - this reflects the individuals satisfying Lowe's unity requirement. It is supposed that the context of an individual can be represented by external relations, e.g. spatial or causal relations - these are represented by the double-headed arrows. 


\subsection{Exemplar metaphysical view of individuals vs. processes found in science}

How well can this exemplar metaphysical view of individuals capture processes found in science, including the range of characterising criteria of such processes identified in Section 2? As outlined in Section 3, to facilitate consideration of this question, the focus is on entities which might be viewed both as processes and individual things.

The exemplar view seems prima facie well-placed to capture how a process is at each stage. Perhaps the having of a part of some type (criterion a), and/or the obtaining of a spatial arrangement of these parts (criterion b), may be taken to be admissible properties. Lewis, however, rejects such structural properties (Lewis 1986), and whilst Armstrong does accept structural properties in a limited sense (Armstrong 1986), his account of such properties cannot capture a distinction between butane and isobutane, for example, which are both $\mathrm{C}^{4} \mathrm{H}^{10}$. So even in capturing the characteristics of a single stage of a process, the exemplar view faces challenges.

How might the exemplar view capture the nature of the change that a process exhibits across certain stages (criterion c)? For processes that are not fully homogeneous (criterion d), doing so will typically require, inter alia, positing a property which captures changing from one state to another state. Consider for example a neuron (which may be viewed from a process perspective as a heterogeneous on-going process) which at some stage has certain parts acting to transmit an action potential. Viewed as an individual thing, it seems we would want to ascribe to this neuron some such property as is-firing. The neuron firing entails, we may suppose, the receipt of neuro-transmitter particles (state A, say) initiating a process which features as a later stage the release of further neuro-transmitter particles (state $B$, say). The putative property is-firing then has the form is-changingfrom-state-A-to-state- $B$ (i.e. is-firing entails the neuron has the property of being about to release neuro-transmitter particles). ${ }^{14}$ But such a property does not obtain before or after such a change, nor can it apply during the change as the change is then incomplete and may not be completed - it seems there is no time at which such a property might obtain. Perhaps we could make do with the less specific property: being-such-as-to-change-from-state-A-to-state- $B$. But here it seems that how the individual changes across stages is ontologically prior to the being of the individual at each stage (at which this putative property obtains), and this might be deemed by some as incompatible with the notion of an individual on their version of the exemplar view. ${ }^{15}$ Another approach might be to associate an individual with its characteristic processual behaviour via a power, e.g. an individual is ascribed the power can-change-from-state-A-to-state-B. Of course, an adoption of powers would

\footnotetext{
${ }^{14}$ I am not considering here behaviours which may be thought continuous or on-going (e.g. rolling, expanding, cooling) - these might perhaps plausibly be taken as characterising how a thing is at some point (or perhaps brief period) of time, and hence be allowed as properties of the thing at that time. ${ }^{15}$ This point echoes the insight of E. J. Lowe that instantaneous velocity might plausibly be a power (and hence a property), but changing location from one place to another cannot. (Lowe 2006, 139-140).
} 
be anathema to many metaphysicians sympathetic to exemplar-like views. In any case, admitting powers may not resolve the problem: if the manifestation of the power is a process, then it seems that processes must be admitted to the ontology after all; and if the manifestation is not a process, then it is not clear that the characteristic of exhibiting processual change has been captured. It seems controversial whether the exemplar view can capture how a process changes across stages.

Kripke, amongst others, proposes that certain properties associated with origin are admissible properties of an individual (Kripke 1980). Perhaps, then, the association of an individual with certain originating-things (criterion e) might count as admissible properties on the exemplar view. But Kripke's view is controversial. And further liberalism would be required to license the inclusion of putative properties which capture the nature of things which leave or join during a process (criterion $f$ ), or which remain at the end of a process (criterion $\mathrm{g}$ ).

Recognising an entity as a process licenses inclusion within our characterisation criteria of how it self-maintains at each stage. By contrast, the representation of change on the exemplar view would seem to be limited to the obtaining of one property at one time and then the obtaining of another different property at a later time. It is not clear how to capture the characteristic form of self-maintenance exhibited by a process, criterion (h), within the exemplar view.

How might we capture the role of a part in a larger process (criterion (i)), and/or the context in which that part occurs (criterion (j)). If this part is taken to be an individual, then the process of which it is a part cannot also be an individual on the exemplar view (due to the unity requirement). It seems the roles and context of a part must be captured by external relations between that part and other parts. These external relations would often need to capture multiple subtle and perhaps intricate roles, and highly complex contexts which are changing over time. Whether sufficient relations of the required kind are admissible is doubtful - even the admissibility of relatively simple spatial and causal relations is controversial.

Again, the exemplar view seems to face challenges in capturing the unity of an individual which has differing phase-stages (criterion $\mathrm{k}$ ) - what is it on this view that unites the differing stages with widely differing properties?

The more liberally we interpret the characteristics of an individual which we can capture within the exemplar list of admissible properties, the more we compromise the parsimonious metaphysical intuitions which motivate such a view initially. Even on the most liberal interpretations of such properties, it is not clear how important aspects of processes can be adequately captured.

This brief investigation suggests that metaphysical views of individuals face challenges in capturing the wide range of characteristics of processes we find reflected in the practice of science. 
The contrast between Figures 5 and 7 highlights pictorially a further important tension between the view of processes in science and the exemplar metaphysical view of individuals: It seems that parts may remain present in the processes of which they are a part, so that both the part and the process (of which it is a part) may then obtain as valid unities (the latter, and perhaps the former, as a process). This contrasts with the exemplar metaphysical view according to which an individual cannot have a part which is also an individual (as represented by the discreteness of the boxes in Figure 7).

It may be that the metaphysical view that either a (possibly complex) whole or a part of that whole may be an individual, but not both, has influenced current debates in the sciences. Questions concerning individuals are often couched in terms of exclusive alternatives within these debates: Is an organism or a species an individual? (Hull 1976, Rieppel 2009.) Is the eukaryotic part of an organism or the symbiotic consortium (with prokaryotic parts) an individual? (Dupré 2012) Is a biofilm or a constituent bacterium an individual? (Ereshefsky \& Pedroso 2016) Is a cell or the organism of which it is a part an individual? (See e.g. Fagan 2016, especially section 7.2.) Figure 5 suggests the way in which a processual view may license a more accommodative answer: a processual unity at one level (pictured as an entwining of strands) does not preclude another differing processual unity at a higher or a lower level.

\section{Conclusion}

A consideration of the scientific treatment of processes suggests that the adequate characterisation of processes requires a rich framework of criteria which may include (in addition to how the process is at each stage), how the process changes and selfmaintains across stages, the nature of the context in which the process typically acts, and the role(s) it plays in this context.

A preliminary and limited comparison of this view of processes distilled from science with metaphysical views of individuals suggests a tension between these views. Metaphysical views often suppose individuals can be wholly characterised by a list of admissible properties, where tight admissibility may be adopted on grounds of parsimony. There is a tension between the adequate characterisation of processes and parsimony of admissible properties. Furthermore, metaphysics often supposes that individuals cannot have parts which are themselves individuals, whereas no such limitation obtains for processes: processes may have parts which are themselves processes. Further articulating, understanding and perhaps resolving these tensions may be some of the work required to develop an adequate account of the individuation of processes. ${ }^{16}$

In the meantime, it seems reasonable to choose to individuate processes in ways which have respect for the rich view of processes reflected in the practice of science,

\footnotetext{
${ }^{16}$ I take up this task in Pemberton (forthcoming).
} 
including the wide range of characterising criteria employed. Using more limited criteria for individuation (e.g. a list of admissible properties), perhaps in deference to a particular metaphysical account of individuals, may be detrimental to the task of individuating processes - perhaps this is one source of the problems concerning individuation which this volume seeks to address. If the suggestion in Section 3 is right that many things may be processes, then the individuation of processes has wide application.

Respecting such a processual view may license recognising both processes and certain of their parts (e.g. organism / cell, biofilm / bacterium, species / organism) as both being valid unities - perhaps robustly self-maintaining processes which can be picked out sufficiently reliably from their natural context. This may provide a basis for the resolution of some important contemporary debates concerning individuation.

\section{Acknowledgment}

For helpful comments and advice on earlier drafts of this paper, I should like to thank Nancy Cartwright, Anna Marmodoro, discussants at a presentation to the Powers Structuralism programme in Oxford (November 2014), various participants at the Taiwan conference on Scientific Individuation (December 2014) and two anonymous referees.

\section{References}

Armstrong, David M. 1986. In defence of structural universals. Australasian Journal of Philosophy 64:1, 85-88.

Bapteste, Eric, and John Dupré. 2013. Towards a processual microbial ontology. Biology \& Philosophy 28: 379-404.

Bear, Mark F., Barry W. Connnors and M. A. Paradiso. 2007. Neuroscience: exploring the brain. Third edition. Baltimore MD: Lippincott, Williams and Wilkins.

Bechtel, William, and Adele Abrahamsen. 2005. Explanation: a mechanist alternative. Studies in the History and Philosophy of the Biological and Biomedical Sciences 36: 421-441.

Cartwright, Nancy. 1999. The dappled world: a study of the boundaries of science. Cambridge: Cambridge University Press.

Cartwright, Nancy and John M. Pemberton. 2012. Aristotelian powers: without them, what would modern science do? In Powers and capacities in philosophy: the new Aristotelianism, ed. John Greco and Ruth Groff. London: Routledge.

Copeland, Robert A. 2000. Enzymes (Second edition). Wiley-VCH, New York. 
Craver, Carl. 2007. Explaining the brain. Oxford: Clarendon Press.

Chen, Ruey-Lin. 2017. Mechanisms, capacities, and nomological machines:

Integrating Cartwright's account of nomological machines and Machamer, Darden and Craver's account of mechanisms. In Philosophy of science in practice, ed. HsiangKe Chao and Julian Reiss. Synthese Library 379: 127-145.

Darden, Lindley. 2006. Reasoning in Biological Discoveries: Essays on Mechanisms, Interfield Relations, and Anomaly Resolution. Cambridge: Cambridge University Press.

Duns Scotus. 1639. Opera omnia. Ed. Luke Wadding. 12 volumes, Lyon, reprinted Hildesheim: Georg Olms Verlagsbuchhandlung, 1968.

Dupré, John. 2010. It is not possible to reduce biological explanations to explanations in chemistry and/or physics. In Contemporary Debates in Philosophy of Biology, ed. Francisco José Ayala and Robert Arp. Malden MA: Wiley-Blackwell Publications.

Dupré, John. 2012. Processes of life. Essays in the philosophy of biology. Oxford and New York: Oxford University Press.

Dupré, John. 2013. Living causes. In Mechanisms and causation in biology. Proceedings of the Aristotelian Society, Supplementary Volume LXXXVII: 19-37.

Eijkelkamp, Niels, John E. Linley, Mark, D. Baker, Michael S. Minett, Roman Cregg, Robert Werdehausen, Francois Rugiero and John N. Wood. 2012. Neurological perspectives on voltage-gated sodium channels. Brain 135(9): 2585-2612.

Ereshefsky, Mark, and Makmiller Pedroso. 2016. What biofilms can teach us about individuality. In Individuals across the sciences, ed. Alexandre Guay and Thomas Pradeu, 103-121. New York: Oxford University Press.

Fagan, Melinda B. 2016. Cell and body. In Individuals across the sciences, ed. Alexandre Guay and Thomas Pradeu, 122-143. New York: Oxford University Press.

Glennan, Stuart. 2002. Rethinking mechanistic explanation. Philosophy of Science 69, S342-353.

Glennan, Stuart. Forthcoming. The new mechanical philosophy. Oxford University Press.

Guay, Alexandre, and Thomas Pradeu. 2016. Individuals across the sciences. New York: Oxford University Press.

Hull, David L. 1976. Are species really individuals? Systematic zoology 25(2), 174-191. 
Illari, Phyllis and Jon Williamson. 2012. What is a mechanism? Thinking about mechanisms across the sciences. European Journal of Philosophy of Science 2: 119135.

Keller, Rebecca W. 2013. Middle school chemistry grades 5-8. Gravitas Publications, Inc.

Kripke, Saul A. 1980. Naming and Necessity, Cambridge, MA: Harvard University Press.

Lewis, David K. 1986. Against structural universals. Australasian Journal of Philosophy 64: 25-46.

Lowe, E. Jonathan. 2006. The four-category ontology. Oxford University Press, USA.

Lowe, E. Jonathan. 2016. Non-individuals. In Individuals across the sciences, ed.

Alexandre Guay and Thomas Pradeu, 46-60. New York: Oxford University Press.

Machamer, Peter, Lindley Darden and Carl F. Craver. 2000. Thinking about mechanisms. Philosophy of science 67: 1-25.

Marcus, Ruth B. 1975. Dispensing with possibilia. Proceedings and Addresses of the American Philosophical Association 49: 39-51.

Marmodoro, Anna. 2013. Aristotle's hylomorphism without reconditioning. Philosophical Inquiry 37 (1-2): 5-22.

McCullough, Laurence B. 1996. Leibniz on individuals and individuation. Springer Science and Business Media, B.V.

Mumford, Stephen and Matthew Tugby, eds. 2013. Metaphysics and science. Oxford: Oxford University Press.

Pemberton, John M. 2011. Integrating mechanist and nomological machine ontologies to make sense of what-how-that evidence (manuscript). http://personal.Ise.ac.uk/pemberto

Pemberton, John M. and Nancy Cartwright. 2014. Ceteris paribus laws need machines to generate them. Erkenntnis 79: 1745-1758.

Pemberton, John M. (Forthcoming). Actor-process ontology.

Quine, Willard V. O. 1948. On what there is. Reprinted in Quine 1953. From a logical point of view. Harvard University Press, Cambridge.

Rieppel, Olivier. 2009. Species as process. Acta Biotheoretica 57(1-2): 33-49. 
Russell, Bertrand. 1913. On the notion of cause. Proceedings of the Aristotelian Society (New series) 13: 1-26.

Russell, Bertrand. 1948. Human knowledge. Routledge, Taylor \& Francis Group, London and New York.

Salmon, Wesley C. 1984. Scientific explanation and the causal structure of the world. Princeton University Press, Oxford.

Scaltsas, Theodore. 1994. Substances \& universals in Aristotle's Metaphysics. Cornell University Press, Ithaca and London.

Sider, Theodore. 2006. Bare particulars. Philosophical Perspectives 20: 387-397.

Südhof, Thomas C. and Josep Rizo. 2011. Synaptic Vesicle Exocytosis. Cold Spring Harbor Perspectives in Biology 3(12): a005637.

van Inwagen, Peter. 1990. Material beings. Cornell University Press, Ithaca and London.

Wu, Cathy H., Hongzhan Huang, Anastasia Nikolskaya, Zhangzhi Hu and Winona C. Barker. 2004. The iProClass integrated database for protein functional analysis. Computational Biology and Chemistry, 28(1): 87-96. 